\title{
A NOTE ON MAXIMUM MODULUS ALGEBRAS
}

\author{
PENTTI JÄRVI
}

1. Let $X$ be a locally compact Hausdorff space and let $A$ be an algebra of complex-valued continuous functions on $X$. Then $A$ is called a (local) maximum modulus algebra on $X$, provided that for each compact subset $K$ of $X$ with topological boundary $\partial K$, and for each $f$ in $A$, we have

$$
\left|f\left(p_{0}\right)\right| \leqq \max \{|f(p)| \mid p \in \partial K\} \text { for } p_{0} \in K
$$

(see [9], [11]). One of the main results of [9] reads as follows ([9, Theorem 2]).

The orem A. If $A$ is a maximum modulus algebra on a plane domain $G$, and if $A$ contains a function which is analytic and not constant in $G$, then every member of $A$ is analytic in $G$.

Recently, Bear and Hile ([2, Theorem 4]) gave the following extension of Theorem A.

Theorem B. Let $G$ be a plane domain and $A$ a maximum modulus algebra on $G$. If for each $z \in G$ there is an open neighborhood $U_{z}$ of $z$ and a function $f_{z} \in A$ such that $f_{z}$ is an interior (i.e., light and open) mapping on $U_{z}$, then there is a homeomorphism $\varphi$ of $G$ onto a plane domain $G^{\prime}$ such that $g \circ \varphi^{-1}$ is analytic in $G^{\prime}$ for each $g \in A$.

In this note we show that the requirement that $f_{z}$ be open is not needed to establish the conclusion of Theorem B; in other words, openness turns out to be a consequence of the remaining assumptions. Actually, our first theorem states that members of any maximum modulus algebra are quasiopen mappings in the sense of Whyburn ([12], [13]); moreover, it is a simple matter to verify that a quasiopen and light mapping is open. Relying on Theorem 1, we will also revise some results of Bear and Hile ([2, Theorem 3]), W. C. Fox ([3]) and Kra ([5, Theorem III]). The note is concluded with some simple observations on algebras of quasiconformal functions (cf. [1]).

2. Let $X$ and $Y$ be topo'ogical spaces. A continuous mapping $f: X \rightarrow Y$ is said to be quasiopen, provided that for any $y \in f(X)$ and any open set $U$ in $X$ containing a compact component of $f^{-1}(y), y$ is an interior point of $f(U)$. The following characterization is due to Whyburn [13, p. 112]. 
Lemma. If $X$ and $Y$ are locally compact Hausdorff spaces, a mapping $f: X \rightarrow Y$ is quasiopen if and only if for each relatively compact open set $U$ in $X \quad \partial f(U) \subset f(\partial U)$.

In fact, Whyburn limited himself to locally compact separable metric spaces, but the validity of the Lemma is readily seen even in the setting given above.

The orem 1. Let $X$ be a locally compact Hausdorff space and let $A$ be a maximum modulus algebra on $X$. Then every member of $A$ is a quasiopen mapping $X \rightarrow C$.

Proof. By [9, Lemma 1], we may assume that $A$ contains the constants. Let $f \in A$ and let $U$ be a relatively compact open set in $X$. Suppose that $\partial f(U) \oplus f(\partial U)$ and take a point $z_{0} \in \partial f(U)$ such that $z_{0} \notin f(\partial U)$. Since $f(\partial U)$ is compact (note that $\partial U$ is nonempty by $(*)$ ), we find a point $z_{1} \in C \backslash f(\bar{U})$ such that $\left|z_{1}-z_{0}\right|<\min \left\{\left|z_{1}-z\right| \mid z \in f(\partial U)\right\}$. Then pick out a point $z_{2} \in f(\bar{U})$ such that $\left|z_{1}-z_{2}\right|=\min \left\{\left|z_{1}-z\right| \mid z \in f(\bar{U})\right\}$. Clearly, $z_{2} \notin f(\partial U)$.

Set $z_{3}=\left(z_{1}+z_{2}\right) / 2$ and

$$
d=\min \left\{\frac{1}{4}\left|z_{1}-z_{2}\right|, \frac{1}{2} \min \left\{\left|z-z_{2}\right| \mid z \in f(\partial U)\right\}\right\} .
$$

Further, let $D\left(z_{2}, d\right)$ stand for the set $\left\{z \in C|| z-z_{2} \mid<d\right\}$. Now choose a point $q \in U$ such that $f(q)=z_{2}$ and denote by $C$ the nonempty compact set $f^{-1}\left(\overline{D\left(z_{2}, d\right)}\right) \cap \bar{U}$. It is clear that $C \subset U$ and $z_{2} \notin f(\partial C)$. Thus $\left|z_{3}-z_{2}\right|<\min \left\{\left|z-z_{3}\right| \mid z \in f(\partial C)\right\}$.

Denote by $g$ the mapping $p \mapsto\left(z_{3}-f(p)\right)^{-1}, p \in C$. By the previous inequality, $(* *)$

$$
|g(q)|>\max \{|g(p)| \mid p \in \partial C\} .
$$

Consider the identity

$$
\left(z_{3}-f(p)\right)^{-1}=\left(z_{3}-z_{2}\right)^{-1} \cdot \sum_{i=0}^{n}\left(\frac{f(p)-z_{2}}{z_{3}-z_{2}}\right)^{i}+\left(\frac{f(p)-z_{2}}{z_{3}-z_{2}}\right)^{n+1} \cdot\left(z_{3}-f(p)\right)^{-1},
$$

$p \in C, n \in N$. Clearly, the function

$$
f_{n}: p \mapsto\left(z_{3}-z_{2}\right)^{-1} \cdot \sum_{i=0}^{n}\left(\frac{f(p)-z_{2}}{z_{3}-z_{2}}\right)^{i}
$$

is a member of $A$ for each $n$. On the other hand,

$$
\left|\left(z_{3}-f(p)\right)^{-1} \cdot\left(\frac{f(p)-z_{2}}{z_{3}-z_{2}}\right)^{n}\right| \leqq \frac{1}{\left|z_{3}-z_{2}\right|} \cdot\left(\frac{1}{2}\right)^{n}
$$

in $C$ for each $n$. It follows that $f_{n} \rightarrow g$ uniformly on $C$. But this implies that $g$ also attains its maximum modulus on $\partial C$, a contradiction to (**).

We conclude that $\partial f(U) \subset f(\partial U)$ for each relatively compact open set $U$ in $X$. The assertion now follows from the preceding Lemma.

Corollary. Let $X$ be a locally compact Hausdorff space and let $A$ be a maximum modulus algebra on $X$. Then $f \in A$ is an open mapping $X \rightarrow C$ whenever $f$ is light. 
Remark. It is clear that for an individual function, in general, validity of the maximum principle does not imply quasiopenness (see also [3]).

3. Our first application provides the generalization of Theorem B mentioned before. Although it is an immediate consequence of Theorem B, in view of Corollary to Theorem 1, we prefer to base the proof on Theorem A and hence reproduce some arguments from [2].

Theorem 2. Let $G$ be a domain in $\hat{\boldsymbol{C}}$, the extended plane, and let $A$ be a maximum modulus algebra on $G$. If for each $z \in G$ there is an open neighborhood $U_{z}$ of $z$ and a function $f_{z} \in A$ such that $f_{z}$ is light on $U_{z}$, then there is a homeomorphism $\Phi$ of $G$ onto a plane domain $G^{\prime}$ such that $g \circ \Phi^{-1}$ is analytic in $G^{\prime}$ for each $g \in A$. Accordingly, the conclusion holds whenever A contains a mapping light on $G$.

Proof. Let $z \in G$, and choose an open neighborhood $U_{z} \subset G$ of $z$ and $f_{z} \in A$ such that $f_{z} \mid U_{z}$ is light. By Corollary to Theorem $1, f_{z} \mid U_{z}$ is interior. By Stoillow's theorem $\left(\left[10\right.\right.$, p. 121]), there is a homeomorphism $\varphi_{z}$ on $U_{z}$ such that $f_{z} \circ \varphi_{z}^{-1}$ is analytic on $\varphi_{z}\left(U_{z}\right)$. Let $A_{z}$ stand for $\left\{g \circ \varphi_{z}^{-1} \mid g \in A\right\}$. Then $A_{z}$ is a maximum modulus algebra on $\varphi_{z}\left(U_{z}\right)$ which contains the nonconstant analytic function $f_{z} \circ \varphi_{z}^{-1}$. By Theorem A, $g \circ \varphi_{z}^{-1}$ is analytic on $\varphi_{z}\left(U_{z}\right)$ for each $g \in A$.

It is now readily verified that $G$ together with the local parameters $\left(U_{z}, \varphi_{z}\right)$, $z \in G$, constitutes a Riemann surface $\tilde{G}$; moreover, the members of $A$ are analytic on $\tilde{G}$. Since $\tilde{G}$ is planar, there is a conformal mapping $\Phi$ of $\tilde{G}$ onto a plane domain $G^{\prime}$. Clearly, $g \circ \Phi^{-1}$ is analytic in $G^{\prime}$ for each $g \in A$.

Example. Define $\varphi: \boldsymbol{C} \rightarrow \boldsymbol{R}, \varphi(z)=\operatorname{Re} z$, and denote by $\boldsymbol{C}(\boldsymbol{R})$ the algebra of all continuous complex-valued functions on $\boldsymbol{R}$. Then $\{g \circ \varphi \mid g \in C(\boldsymbol{R})\}$ is a maximum modulus algebra on $C$. This simple example shows that lightness, or something like that, is really needed to guarantee some sort of analyticity.

Next consider the situation of [2, Theorem 3]. In other words, let $G \subset \hat{C}$ be a domain and $A$ a uniform algebra on $\bar{G}$ such that the maximal ideal space of $A$ is $\bar{G}$ and the Shilov boundary $\Gamma$ is a proper subset of $\bar{G}$ (for the terminology, we refer to [4]).

Let $z \in G \backslash \Gamma$ and let $U_{z} \subset G \backslash \Gamma$ be a connected open neighborhood of $z$. Suppose that there is a function $f \in A$ such that $f \mid U_{z}$ is light. Since $A_{z}$, the restriction of $A$ to $U_{z}$, is a maximum modulus algebra on $U_{z}$ by Rossi's theorem [4, p. 92], there is, by Theorem 2, a homeomorphism $\varphi_{z}$ on $U_{z}$ such that $g \circ \varphi_{z}^{-1}$ is analytic on $\varphi_{z}\left(U_{z}\right)$ for each $g \in A$.

As before, the local parameters $\left(U_{z}, \varphi_{z}\right)$ are compatible in an obvious way. Consequently, for each component $D$ of $G \backslash \Gamma$ there is a homeomorphism $\Phi$ of $D$ onto a plane domain such that $g \circ \Phi^{-1}$ is analytic for each $g \in A$. Thus the property of being countable-to-one in the version of Bear and Hile is replaced by lightness in 
Theorem 3. Let $G \subset \hat{C}$ be a domain and $A$ a function algebra on $\bar{G}$. Assume that the maximal ideal space of $A$ is $\bar{G}$ and the Shilov boundary $\Gamma$ is a proper subset of $\bar{G}$. If for each $z \in G \backslash \Gamma$ there is a neighborhood $U_{z}$ of $z$ and a function $f_{z} \in A$ such that $f_{z} \mid U_{z}$ is light, then there is on each component of $G \backslash \Gamma$ a homeomorphism $\Phi$ onto a plane domain such that $g \circ \Phi^{-1}$ is analytic for each $g \in A$.

Remarks. (1) The assumptions of [2, Theorem 1] admit a similar relaxation. This follows immediately from Corollary to Theorem 1, in view of Rossi's theorem.

(2) Apparently, a result analogous to Theorem 3 can be obtained whenever $G$ is a relatively compact domain in any Riemann surface. Similarly, in Theorem 2 $G$ could be taken as an arbitrary Riemann surface.

In a similar fashion, we can establish the following extension of a result of W. C. Fox (see [3]).

Theorem 4. Let $X$ be a topological manifold of dimension two, and let $f$ and $g$ be functions, not both constants, sending $X$ into $C$. There exists a conformal structure for $X$ relative to which both $f$ and $g$ are analytic if and only if the algebra generated by $f$ and $g$ is a maximum modulus algebra on $X$ and at least one member in this algebra is also light.

The next theorem generalizes a striking result of Kra ([5], [7]).

Theorem 5. Let $X$ be a connected, locally compact, Hausdorff space, and let $A$ be a maximum modulus algebra on $X$ which separates points and contains the constants. Suppose that, for every $p \in X$, the ideal $M(p)=\{f \in A \mid f(p)=0\}$ is principal. Then $X$ can be given a unique conformal structure which respects the topology such that every $f \in A$ becomes an analytic function on $X$. In particular, $X$ is an open Riemann surface.

Remark. In Kra's version, the nonconstant functions in $A$ were assumed to be open mappings. Cf. also Remarks (2) and (3) in [5, p. 239].

Proof (as in [7]). Let $p \in X$ and let $t \in A$ be a function which generates $M(p)$. Since $A$ separates points, $t(q) \neq 0$ for each $q \neq p$ in $X$. Let $V$ be an open neighborhood of $p$ with compact closure $\bar{V}$, and denote $\delta=\min \{\mid t(q) \| q \in \partial V\}$ (again, $\partial V \neq \emptyset$ by $(*))$.

Given any $f \in M(p),|f|$ and $|f| t \mid$ attain their maxima for $\bar{V}$ at points on $\partial V$. Hence

$$
\|f / t\| \leqq(1 / \delta) \cdot\|f\|, \quad f \in M(p)
$$

where \|\| refers to the sup norm on $\bar{V}$.

Thus the assumptions of the lemma of Porcelli and Connell (see [7, pp. 318-319]) are satisfied. Consequently, on the open set $U=\{q \in V|| t(q) \mid<\delta / 2\}$, every function $f \in A$ is equal to a convergent power series in $t$; i.e., $f \mid U=g \circ(t \mid U)$, where $g$ is analytic on $\{z \in C|| z \mid<\delta / 2\}$. Since $A$ separates points, $t$ must be injective on $U$. 
It follows from Corollary to Theorem 1 that $t$ is also open on $U$. Accordingly, $t \mid U$ is a homeomorphism of $U$ onto $t(U)=\{z \in C|| z \mid<\delta / 2\}$.

Now, clearly, the pairs $(U, t \mid U)$ constitute a unique conformal structure on $X$ in such a way that each member of $A$ becomes an analytic function on $X$.

4. For the sake of illustration, we will add some observations on $Q C(G)$, the class of quasiconformal functions on a plane domain $G$. Recall that a quasiconformal function on $G$ can be defined as a function $f$ which admits a representation $f=g \circ \varphi$, where $\varphi$ is a quasiconformal homeomorphism $G \rightarrow \varphi(G)$ and $g$ is an analytic function on $\varphi(G)$; thus we include the constants but exclude functions with "poles" (cf. [6, p. 250]).

Assume that $A \subset Q C(G)$ is a nontrivial algebra with the usual operations. By Theorem B, we find a homeomorphism $\varphi: G \rightarrow \varphi(G)$ and an algebra, say $B$, of analytic functions on $\varphi(G)$ such that $A=B \circ \varphi=\{g \circ \varphi \mid g \in B\}$. Plainly, $\varphi$ is quasiconformal on $G$. Thus $A \subset Q C(G)$ constitutes an algebra if and only if there is a quasiconformal homeomorphism $\varphi$ on $G$ and an algebra $B$ of analytic functions on $\varphi(G)$ such that $A=B \circ \varphi$. In particular, the complex dilatations (see [6, pp.191192]) of any two nonconstant members of an algebra coincide (as elements of $L^{\infty}(G)$, of course).

Assume now that $A \subset Q C(G)$ is a maximal algebra, i.e., $A=A^{\prime}$ whenever $A^{\prime}$ is an algebra such that $A \subset A^{\prime} \subset Q C(G)$. Then clearly $A=H\left(G^{\prime}\right) \circ \varphi$, where $H\left(G^{\prime}\right)$ stands for the algebra of all analytic functions on $G^{\prime}=\varphi(G)$. Obviously, there is a one-to-one correspondence between the class of maximal algebras in $Q C(G)$ and the set $\left\{\mu \in L^{\infty}(G) \mid\|\mu\|<1\right\}$ (see [6, p. 204]).

Assume then that $A_{i} \subset Q C(G)$ is a maximal algebra and $\varphi_{i}$ a corresponding quasiconformal homeomorphism, $i=1,2$. Suppose that $T: A_{1} \rightarrow A_{2}$ is an algebraic homomorphism. Let $\varphi_{i}^{*}$ denote the homomorphism $g \mapsto g \circ \varphi_{i}, H\left(\varphi_{i}(G)\right) \rightarrow A_{i}$, $i=1,2$. Then $T^{\prime}=\varphi_{2}^{*-1} \circ T \circ \varphi_{1}^{*}$ is an algebraic homomorphism $H\left(\varphi_{1}(G)\right) \rightarrow$ $H\left(\varphi_{2}(G)\right)$. By [8, Theorem 1], there is a unique analytic mapping $\psi$ of $\varphi_{2}(G)$ into $\varphi_{1}(G)$ such that $T^{\prime} g=g \circ \psi$ for each $g \in H\left(\varphi_{1}(G)\right)$. Consequently, there is a oneto-one correspondence between the class of homomorphisms $T: A_{1} \rightarrow A_{2}$ and the class of analytic mappings $\psi: \varphi_{2}(G) \rightarrow \varphi_{1}(G)$. In particular, $A_{1}$ and $A_{2}$ are algebraically isomorphic if and only if $\varphi_{1}(G)$ and $\varphi_{2}(G)$ are conformally equivalent. 


\section{References}

[1] Bear, H. S., and G. N. HiLe: Algebras which satisfy a second order linear partial differential equation. - Pacific J. Math. 75, 1978, 21-36.

[2] Bear, H. S., and G. N. Hile: Analytic structure in function algebras. - Houston J. Math. 5, 1979, 21-28.

[3] Fox, W. C.: Analyticity without analysis. - Proc. Amer. Math. Soc. 13, 1962, 274-275.

[4] Gamelin, T. W.: Uniform algebras. - Prentice-Hall, Inc., Englewood Cliffs, N. J., 1969.

[5] KRA, I.: On the ring of holomorphic functions on an open Riemann surface. - Trans. Amer. Math. Soc. 132, 1968, 231-244.

[6] Lehto, O., und K. I. Virtanen: Quasikonforme Abbildungen. - Die Grundlehren der mathematischen Wissenschaften 126, Springer-Verlag, Berlin-Heidelberg-New York, 1965.

[7] Richards, I.: Axioms for analytic functions. - Adv. in Math. 5, 1970, 311-338.

[8] Royden, H. L.: Rings of analytic and meromorphic functions. - Trans. Amer. Math. Soc. $83,1956,269-276$.

[9] Rudin, W.: Analyticity, and the maximum modulus principle. - Duke Math. J. 20, 1953, $449-457$.

[10] StoÏLow, S.: Leçons sur les principes topologiques de la théorie des fonctions analytiques. Gauthier-Villars, Paris, 1956.

[11] Wermer, J.: Maximum modulus algebras and singularity sets. - Proc. Roy. Soc. Edinburgh Sect. A $86,1980,327-331$.

[12] Whyburn, G. T.: Quasi-open mappings. - Rev. Roumaine Math. Pures Appl. 2, 1957, 47-52.

[13] Whyburn, G. T.: Topological analysis. - Princeton University Press, Princeton, New Jersey, 1958.

University of Helsinki

Department of Mathematics

SF-00100 Helsinki 10

Finland

Received 16 January 1981 